\title{
Short-range interactions between strongly nonlocal spatial solitons
}

\author{
Wei $\mathrm{Hu}$ * Shigen Ouyang. 7 Pingbao Yang, and Qi Gud \\ Laboratory of Photonic Information Technology, South China Normal University, Guangzhou 510631, P. R. China and \\ Laboratory of Light Transmission Optics, South China Normal University, Guangzhou 510631, P. R. China
}

(Dated: July 23, 2018)

\begin{abstract}
A novel phenomenon is discovered that the short-range interaction between strongly nonlocal spatial solitons depends sinusoidally on their phase difference. The two neighbouring solitons at close proximate can be inter-trapped via the strong nonlocality, and propagate together as a whole. The trajectory of the propagation is a straight line with its slope controlled by the phase difference. The experimental results carried out in nematic liquid crystals agree quantitatively with the prediction. Our study suggests that the phenomenon to steer optical beams by controlling the phase difference could be used in all-optical information processing.
\end{abstract}

Solitons are a common phenomenon appearing in many physical fields, while the interactions of the solitons have great potential for much wild applications. [1] The strongly nonlocal spatial soliton, which is also known the accessible soliton 2] and is the self-trapped optical beam from the balance between diffraction and nonlinearity propagating in nonlocal nonlinear media under the condition of the strong nonlocality [3], has some significative properties and has attracted more attentions in the last decade 2, 4, 5, 6]. Several strongly nonlocal, referred also as highly nonlocal in some literatures (for example, Refs. 2, 4, 5), media have been found in experiments, such as the nematic liquid crystal [4, 5, , 6, 7], the lead glass [8], and the thermal nonlinear liquid 9 , and the nonlinear gas of ions [10].

Nonlocality of nonlinear response may drastically modify the properties of the solitons, specially their interactions. In a strongly nonlocal case, it has been shown theoretically and experimentally that the attraction can occur between the bright solitons with any phase difference [2, 11, 12, 13], the coherent or incoherent solitons [14, 15], or the dark solitons 9, 16]. In the contrary, however, the interaction can be attractive only for two in-phase local bright solitons [1]. Both of the longrange interaction [17] and the short-range interaction between the solitons can happen in strongly nonlocal nonlinearity, while only the short-range interaction can occur in local nonlinearity because the force between the local solitons decreases exponentially with the separation between them 18].

The fact well-known so far was that [2, 11, 12, 13, 17] the interactions between the strongly nonlocal bright solitons are independent of their phase difference. In this letter, we differentiate the patterns of the short-range interaction and the long-range interaction between the strongly nonlocal bright solitons. We show theoretically and experimentally that the short-range interaction of the two strongly nonlocal bright solitons is sinusoidally dependent on their phase difference.

Let us consider a (1+2)-D model of an optical field that polarizes linearly with an envelope $A$ and propagates in $z$-direction in the medium with nonlocal nonlinearity:

$$
2 i k \partial_{z} A+\nabla_{\perp}^{2} A+2 k^{2} \frac{\Delta n}{n_{0}} A=0,
$$

where $\nabla_{\perp}^{2}=\partial_{x}^{2}+\partial_{y}^{2}, k$ and $n_{0}$ are the wave-vector and linear refractive index of the medium. The nonlinear perturbation of refraction index $\Delta n(x, y, z)$ can be generally expressed as

$$
\Delta n=n_{2} \int_{-\infty}^{\infty} R\left(x-x^{\prime}, y-y^{\prime}\right)\left|A\left(x^{\prime}, y^{\prime}, z\right)\right|^{2} d x^{\prime} d y^{\prime},
$$

where $R(x, y)$ is the real nonlinear response function of the medium. The normalized condition, $\int_{-\infty}^{\infty} R(x, y) d x d y=1$, is chosen physically to make the nonlinear index $n_{2}$ have the same dimensions as that in a local Kerr-medium. If $R(x, y)$ is a delta function, $\Delta n=n_{2}|A|^{2}$ and Eq. (11) becomes the well-known nonlinear schrödinger equations (NLSE) for the local Kerrmedium. The Eq.(11) and Eq.(2), so-called the nonlocal nonlinear schrödinger equation (NNLSE), can model the beam propagation in most of nonlocally nonlinear media discovered in experiments so far.

For the NNLSE, several well-known invariant integrals are important for the theoretical analysis [12, 19]. The first one is the power integral, $P=\int_{-\infty}^{\infty}|A(x, y)|^{2} d x d y$, which results from the energy conservation of an optical beam during the propagation in a lossless medium. The second is the momentum [12, 19]

$$
\vec{M}=\frac{1}{k} \int_{-\infty}^{\infty} A^{*}\left(-i \nabla_{\perp}\right) A \mathrm{~d} x \mathrm{~d} y .
$$

The momentum governs the movement of the mass center of the two optical beams, i.e.

$$
\frac{\partial \vec{r}_{c}(z)}{\partial z}=\frac{\vec{M}}{P}
$$

where the mass center $\vec{r}_{c}$ is $\vec{r}_{c}(z)=$ $(1 / P) \int \vec{r}|A(x, y, z)|^{2} d x d y$, and $\vec{r}=x \hat{e}_{x}+y \hat{e}_{y}$. The trajectory of mass center is a straight line with its slope determined by Eq. (4). 

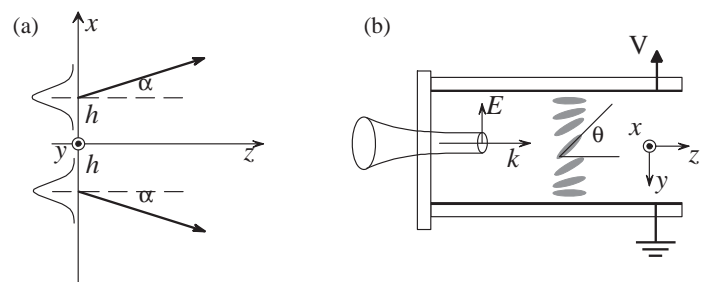

(c)

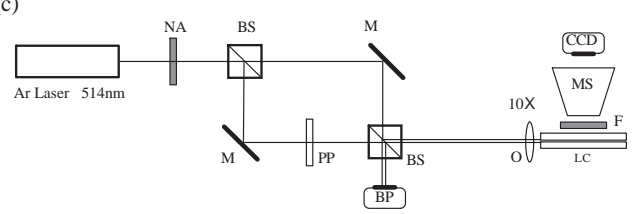

FIG. 1: The sketchs of (a) the two injective solitons, (b) the liquid crystal cell, and (c) the experimental setup. NA, neutral attenuator; BS, beam splitters; M, plate mirror; PP, parallel-face plate for adjusting the phase difference; $\mathrm{O}, 10 \times$ microscope objective; LC, liquid crystal cell; MS, microscope; $\mathrm{F}$, laser-line filter; BP, beam profiler.
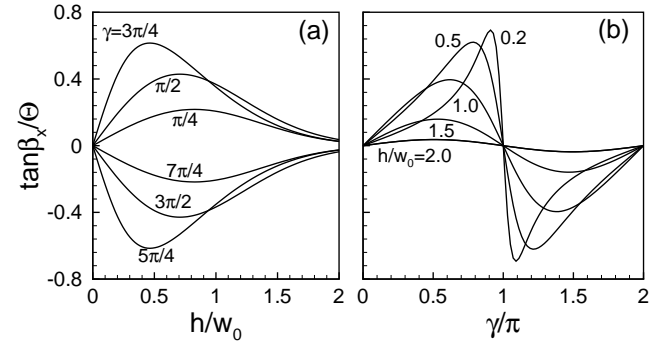

FIG. 2: The dependence of the slope on the distance $h$ (a) and phase difference $\gamma$ (b) for two parallel-injected solitons.

Suppose the two simultaneously incident Gaussian solitons are coplanar in $x-z$ plane, with a width $w_{0}$, a phase difference $\gamma$ and a separation $d(=2 h)$, as shown in Fig. 1(a), i.e.

$$
\begin{aligned}
A(x, y, 0) & =A_{0} \exp \left[-\frac{(x+h)^{2}+y^{2}}{2 w_{0}^{2}}+i k(x+h) \tan \alpha\right] \\
& +A_{0} e^{i \gamma} \exp \left[-\frac{(x-h)^{2}+y^{2}}{2 w_{0}^{2}}-i k(x-h) \tan \alpha(5)\right.
\end{aligned}
$$

where the amplitude $A_{0}$ is large enough to make the two beams propagate in soliton states[2, 4, 5, 11]. For the input condition (5), the slope can be obtained

$$
\frac{\tan \beta_{x}}{\Theta}=\frac{\left(h / w_{0}\right) \exp \left[-\left(h / w_{0}\right)^{2}-(\tan \alpha / \Theta)^{2}\right] \sin \gamma}{1+\exp \left[-\left(h / w_{0}\right)^{2}-(\tan \alpha / \Theta)^{2}\right] \cos \gamma}
$$

and $\tan \beta_{y}=0$, where $\tan \beta_{x}$ and $\tan \beta_{y}$ are the slops in $x-z$ and $y-z$ planes respectively, and $\Theta=1 / k w_{0}$ is the far-fields divergence angle of a Gaussian beam.

The slope of the line for the trajectory of mass center is greatly dependent on the separation $d$ and the phase

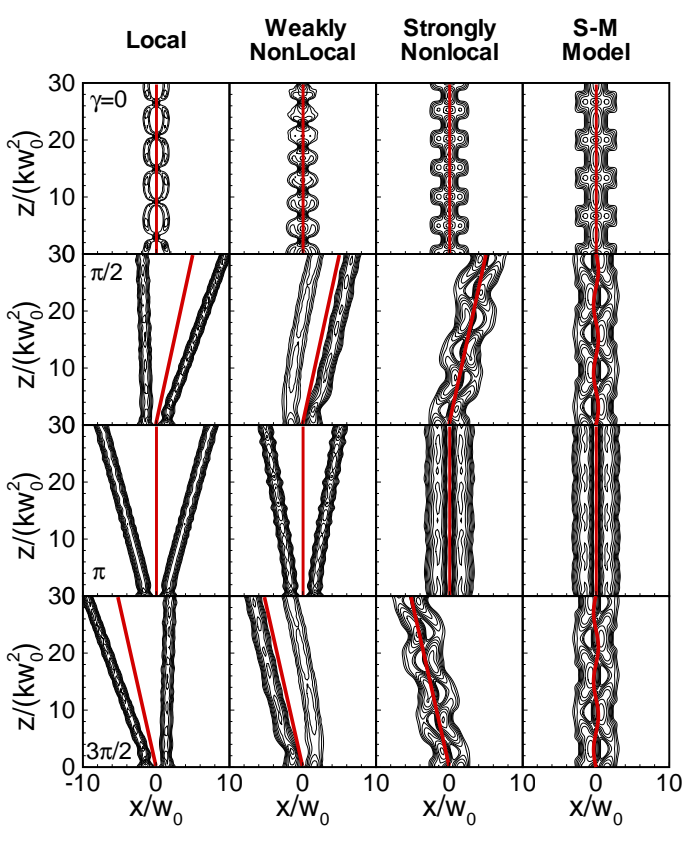

FIG. 3: The contour graph of the numerical propagation of Eqs. (1) and (2) for the two parallel-injected solitons. The local case is shown in the first (from the left to the right) column, and the two different nonlocal cases with an exponentialdecay response function given by Eq. (17) are in the second and third columns, respectively. The red solid lines show the movement of the mass center of the two solitons. The results from the Snyder-Mitchell model[2] with the same initial condition are also given in the fourth column for comparison. The phase difference between two solitons are $0, \pi / 2, \pi$, and $3 \pi / 2$, respectively (from the top to the bottom).

difference $\gamma$, as shown in Fig. 2, where we take $\alpha=0$, that is, two solitons are parallel injected into the medium. Figure 2 shows that $\tan \beta_{x}=0$ only when $\gamma=0$ or $\pi$ for $h / w_{0} \leq 2$, and $\tan \beta_{x}$ goes toward to zero when $h / w_{0} \geq 2 . \tan \beta_{x}$ has significant value when $h$ is about or smaller than the beam width $w_{0}$. In other words, when the soliton separation $d$ is approximate or larger than four times the soliton width $w_{0}$, the optical fields of the two solitons do not overlap so that $\tan \beta_{x}$ decreases to zero, otherwise the two solitons are of an effective overlap and $\tan \beta_{x}$ has a non-zero value changeable by the phase difference.

It is important to emphasize that the above analytical result about the movement of the mass center [Eq. (6)] is universal, independent of the form of nonlinear response function $R$. This means that no matter what the material is and how the degree of nonlocality is, the movement of the mass center is the same for the initial condition (5).

Although the momentum gives the movement of the mass center, it is difficult to obtain the analytical solution of the beam propagation for the initial condition (5). We carry out the numerical simulation for local, weakly nonlocal, and strongly nonlocal propagations, re- 
spectively, and only the (1+1)-D case of Eqs. (1) and (2) is simulated for the sake of simplicity and also without the loss of generality. The $(1+1)$-D model makes it possible to compare the propagations in the nonlocal nonlinearity and in the local nonlinearity, and also provides an exact enough description to the $(1+2)$-D coplanar propagation. The results are shown in Fig. 3 The nematic liquid crystal (NLC) is taken into consideration here for the consistency with our experiment discussed later in this letter. The response function of the NLC is the exponential-decay function

$$
R(x)=\left(1 / 2 w_{m}\right) \exp \left(-|x| / w_{w}\right),
$$

for the (1+1)-D case 12], and the zero-th order modified Bessel function, $R(x, y)=\left(1 / 2 \pi w_{m}^{2}\right) K_{0}\left(\sqrt{x^{2}+y^{2}} / w_{m}\right)$, for the (1+2)-D cylindrical symmetrical case [4, 13], where $w_{m}$ is the bias-voltage controllable characteristic length 13 of the response function $R$ for the NLC. The ratio $w_{m} / w_{0}$ indicates the degree of nonlocality [3] , which are chosen in simulation to be 0.47 and 10 for weakly nonlocal and strongly nonlocal cases in Fig. 3. respectively.

Some interesting consequences can be obtained from Fig. 3. For the local system described by the NLSE, the two solitons attract each other only for the in-phase case $(\gamma=0)$ and repel each other for other occasions [1]. There also exists the power transfer between the solitons when $\gamma$ is not equal 0 and $\pi$, as mentioned in Ref. [1]. The force between the in-phase solitons is always attractive, independent of the degree of nonlocality, as shown in the first row; while the repelling force between the solitons for the other phase difference cases becomes weaker, as the degree of nonlocality increases. As a result, the two solitons with an arbitrary phase difference can get attractive when nonlocality becomes strong enough. For all of those propagations, however, the movement of the mass center obeys the same regulation, a straight-line trajectory with its slope given by Eq. (6). It is clear that the strong enough nonlocality can make the two spatial solitons trap each other and propagate together as a whole, going along the mass center trajectory, the straight line, with the slope steered by their initial phase difference. Interestingly, a similar phenomenon was mentioned 20] in the intermediate process 21] for dealing with incoherent solitons in "fast" nonlocal nonlinear media.

It can be observed that the maximum value of the tilting angles occurs when $\gamma$ approximates $\pi$ for small distance $h$. For each $h$, two values $\gamma_{\max }$ between $\pi / 2$ and $3 \pi / 2$, which is determined by $\cos \gamma_{\max }=$ $\pm \exp \left(-h^{2} / w_{0}^{2}\right)$, makes $\beta_{x}$ reach the extrema (a maximum and a minimum, respectively), $\tan \left[\left(\beta_{x}\right)_{\text {ext }}\right] / \Theta=$ $\pm\left(h / w_{0}\right) \exp \left(-h^{2} / w_{0}^{2}\right) / \sqrt{1-\exp \left(-2 h^{2} / w_{0}^{2}\right)}$. The maximum is for steering right and the minimum for left. The smaller $h$, the larger tilt angle $\beta_{x}$. The largest tilting angle is $\Theta / \sqrt{2}$. It means the steering angle of whole beam are significance only for thin beams.
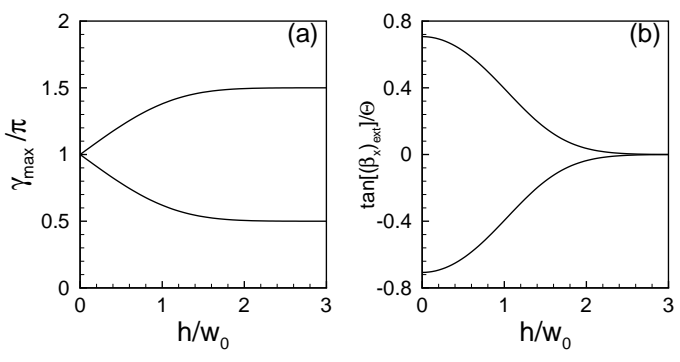

FIG. 4: (a) the phase difference $\gamma_{\max }$ for the maximum slope angle vs the distance $h$, and (b) the maximum of tilt angle $\tan \left[\left(\beta_{x}\right)_{\text {ext }}\right]$ vs the distance $h$. Both are for $\alpha=0$.

Togetherness of Figs. 2] and 3 can result in one more significant outcome. When $d<4 w_{0}$, the two solitons have a non-zero overlap and the slope $\tan \beta_{x}$ is no-zero also. In this case, two solitons can be inter-trapped via the strong nonlocality, and propagate together as a whole, going along the diagonal line of the trajectory for their mass center. This is the short-range interaction between the strongly nonlocal solitons, which is phase-sensitive (controllable by their phase difference). When $d>4 w_{0}$, on the other hand, the two solitons never overlap and the slope of the trajectory for their mass center tends toward zero. In this case, two strongly nonlocal solitons undergo periodic collisions in the coplanar propagation [2, 11, 17], and spiral about one another if they are initially skew to each other 2, 17]. Both processes have nothing to do with their relative phase, as predicted first by Snyder and Mitchell [2] and verified experimentally 11, 17]. This is the long-range interaction between the strongly nonlocal solitons. As the soliton separation $d$ increases from less to larger than $4 w_{0}$, the interaction will gradually transit from the short-range pattern to the long-range one in the strongly nonlocal nonlinearity, and vice versa. Only the short-range interaction, however, exists in the local nonlinearity [18].

Two more points are given in the end of the theoretical part. It is worth to note that the Snyder-Mitchell model 2] can not give right prediction about the shortrange interaction between the strongly nonlocal solitons, as shown in the fourth column of Fig. 3. Such a phasecontrollable short-range interaction might have its application in all-optical switching and routing.

In order to verify our prediction, we carried out the experiment in the NLC.

The configuration of the NLC cell is the same as in the previous works [5, 7, 13], as shown in Fig. 1(b). The optical field polarized in $y$-axis with envelope $A$ propagates in $z$-direction. An external low-frequency electric field $E_{R F}$ is applied in $y$-direction to control the initial tilt angle of the NLC.

The experimental setup is illustrated in Fig. 1(c). The laser beam from the laser is split into two beams, then they are combined together with a small separa- 
tion through the other beam-splitter and launched into a $80 \mu m$-thick NLC cell by a $10 \times$ microscope objective. The beam width at the focus $w_{0}$, the separation $d$, and relative angle $2 \alpha$ between the two beams are measured by an edged-scanning beam profiler when the NLC cell is removed. The cell is filled with the NLC TEB30A (from SLICHEM China Ltd.), whose $n_{\|}=1.6924, n_{\perp}=$ $1.5221, K \approx 10^{-11} N, \epsilon_{a}^{o p}=0.5474$, and $\epsilon_{a}^{R F}=9.4$. The bias voltage on the cell is set to $1.4 \mathrm{~V}$, and then a pretilt angle is nearly $\pi / 4$ in order to obtain strong enough nonlocality and the lowest critical power of solitons [13]. The launched power for each beam is fixed to $6 \mathrm{~mW}$, and two spatial solitons are obtained for such high enough excitations. The parameters for the beams inside the NLC are calculated from the measurement without NLC cell, i.e. $w_{0}=2.2 \mu \mathrm{m}, d=2.25 \mu \mathrm{m}, \tan (2 \alpha)=0.0076$, and the divergence angle $\Theta=0.0231$.

The phase difference between the two beams (solitons) is adjusted by the rotation of a $1.8 \mathrm{~mm}$-thick parallelface plate, and measured through the interference pattern by the beam profiler located on the other branch after the second beam-splitter. First we find the position of the plate while the phase difference is adjusted to 0 (in phase), then we rotate the plate in small steps to increase the phase difference $\gamma$.

We record the soliton trajectories for the different situations by the CCD camera, as shown in Fig.5. In Fig. 5 (a) and (b), each of two solitons is alone launched into the NLC respectively, and their trajectories are straight and horizontal. When two solitons are injected simultaneously into the NLC, they will propagate as a whole, and tilt (c) up or (d) down (actually in $x$-direction). Since the separation is so small that two solitons cannot be distinguished by the microscope in our experiment, we will see a whole beam, as a bound state, steered by the phase difference $\gamma$.

In order to compare quantitatively our experimental observation with our theoretical prediction, we give the variation of the tilting angle with the phase difference $\gamma$ in Fig. 6. For each $\gamma$, we take five photos of the beam tilting angles to minimize the jitter of the tilting angle resulting from that of the laser source and the phase difference. We can see that the experiment points locate around the theoretical prediction with a relative small random error. The error may mainly come from the slight jitter of the phase difference $\gamma$. Except those random error, we can say that the experiment results consist with the theoretical prediction very well. The maximum tilt angle observed in experiment is about $1.2^{\circ}$, approximate $0.6 \Theta$.

In conclusion, we predict that the short-range interaction between the strongly nonlocal solitons depends drastically on their phase difference. The result is universal, independent of the different nonlocal nonlinear media. The experiment carried out in the nematic liquid crystal agrees quantitatively with the prediction. The SnyderMitchell model can only give the right prediction for the

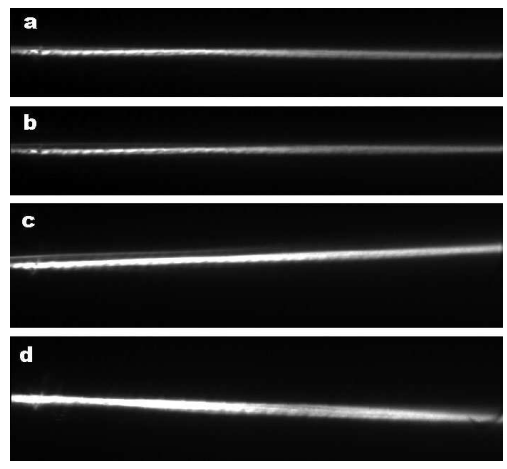

FIG. 5: Photos of the beam trajectories for the single soliton $[(a)$ and (b)] and the two solitons injected together [(c) and $(\mathrm{d})]$ propagating in the NLC cell. The phase difference between the two solitons for (c) and (d) are about $\pi / 2$ and $3 \pi / 2$, respectively.

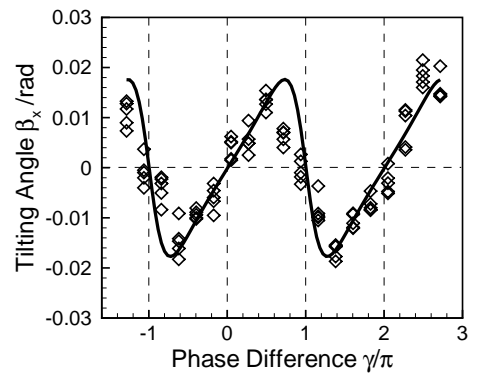

FIG. 6: The tilting angle of two beams vs. the phase difference between them. Square points: experiment results, solid curve: the theoretical fitting curve from Eq. (6).

long-range interaction between the strongly nonlocal solitons.

Unlike their local counterpart, the strongly nonlocal solitons can exhibit both the short-range interaction and the long-range interaction. The two kinds of interactions, however, have different patterns, the former is phasesensitive and the latter is not. Therefore, each of the two phenomena would provide a means of controlling light with light, and be thus potentially useful in developing all-optical signal processing devices. They are, of course, supposed to be applied in different situations.

This work was supported by the National Natural Science Foundation of China (Grant No.10674050), and partially supported by the National Natural Science Foundation of China (Grant Nos. 10474023 and 60278013). The authors would like to thank Prof. Li Xuan for her supply of the NLC cell samples.

* These authors contributed equally to this work. $\dagger$ guoq@scnu.edu.cn 
[1] G. I. Stegeman and M. Segev, Science 286, 1518 (1999) and references therein.

[2] A. W. Snyder and D. J. Mitchell, Science 276, 1538 (1997).

[3] W. Krolikowski and O. Bang, Phys. Rev. E 63016610 (2001); Q. Guo, B. Luo, and S. Chi, Opt. Commun. 259, 336 (2006); Q. Guo, B. Luo, F. Yi, S. Chi, and Y. Xie, Phys. Rev. E 69, 016602 (2004).

[4] C. Conti, M. Peccianti, and G. Assanto, Phys. Rev. Lett. 91, 073901 (2003).

[5] C. Conti, M. Peccianti, and G. Assanto, Phys. Rev. Lett. 94, 113902 (2004).

[6] M. Peccianti, C. Conti, G. Assanto, A. De Luca, and C. Umeton, Nature, 432, 733 (2004).

[7] M. Peccianti, A. De Rossi, G. Assanto, A. De Luca, C. Umeton, and I. C. Khoo, Appl. Phys. Lett. 77, 7 (2000).

[8] C. Rotschild, O. Cohen, O. Manela, M. Segev, T. Carmon, Phys. Rev. Lett. 95, 213904 (2005)

[9] A. Dreischuh, D. Neshev, D. E. Peterson, O. Bang, W. Królikowski Phys. Rev. Lett. 96, 043901(2006)

[10] D. Suter, and T. Blasberg, Phys. Rev. A 45, 4583 (1993).

[11] M. Peccianti, K. Brzdakiewicz, and G. Assanto, Opt. Lett. 27, 1460 (2002).

[12] P. D. Rasmussen, O. Bang, and Wieslaw Królikowski, Phys. Rev. E 72, 066611 (2005).

[13] W. Hu, T. Zhang, Q. Guo, L. Xuan, S. Lan, Appl. Phys.
Lett. 89, 071111 (2006).

[14] M. Peccianti,and G. Assanto, Phys. Rev. E 65, 035603(R) (2003).

[15] M. Shen, Q. Wang, J. Shi, Y. Chen, X. Wang, Phys. Rev. E 72, 026604(2005).

[16] N. I. Nekolov, Opt. Lett. 29, 286(2004).

[17] C. Rotschild, B. Alfassi, O. Cohen and M. Segev, Nature Physics, 2, 769 (2006).

[18] J. P. Gordon, Opt. Lett. 8, 596 (1983)

[19] A. I. Yakimenko, V. M. Lashkin, and O. O. Prikhodko, Phys. Rev. E 73, 066605 (2006).

[20] O. Cohen, H. Buljan, T. Schwartz, J. W. Fleischer, and M. Segev, Phys. Rev. E 73, 015601(R) (2006).

[21] In the Ref. 20, the propagation of incoherent solitons in "fast" nonlocal nonlinear media was studied in two steps: (1) analyzing the propagation within a very short time interval (a time frame much shorter than the characteristic fluctuation time) during which the beam can be treated as a coherent speckled wave, and (2) calculating the propagation of the time-averaged envelope. In the intermediate process (step 1), a similiar phenomenon to steer self-trapped propagation by control their phase difference is found to be exist for two overlap beams. After step 2, however, such phase sensitivity is lost for the time averaged envelope that is a really observed result. 